\title{
Fixed Drug Eruption
}

National Cancer Institute

\section{Source}

National Cancer Institute. Fixed Drug Eruption. NCI Thesaurus. Code C111986.

Round areas of red-purple reaction in the skin that result after drug exposure; these recur in the same location when the medication is readministered. 